\title{
Heavy quark pair production in proton-proton collisions including subdominant terms
}

\author{
Marta Łuszczak* \\ University of Rzeszow, Poland \\ E-mail: luszczak@univ.rzeszow.pl
}

\section{Antoni Szczurek}

University of Rzeszow \& Institute of Nuclear Physics PAN, Poland

E-mail: Antoni.Szczurek@ifj.edu.pl

\begin{abstract}
Gluon-gluon fusion is the dominant process of heavy quark production in high-energy protonproton collisions. The corresponding calculations were performed in the standard collinear approach as well $\mathrm{k}_{t}$-factorization approach based on unintegrated parton distributions functions (UGDFs). We have tested several models of UGDFs. Here we concentrate on subdominant contributions initiated by photons ignored so far in the literature. Both elastic and inelastic processes have been considered. We discuss processes initiated by $\gamma g \rightarrow Q \bar{Q}, g \gamma \rightarrow Q \bar{Q}, \gamma \gamma \rightarrow Q \bar{Q}$ subprocesses. In this context we use MRST-QED (Martin, Roberts, Stirling, Thorne) parton distribution set which includes also photon as a parton of the proton. The subleading processes considered here are shown in Fig.1
\end{abstract}

35th International Conference of High Energy Physics

July 22-28, 2010

Paris, France

${ }^{*}$ Speaker. 


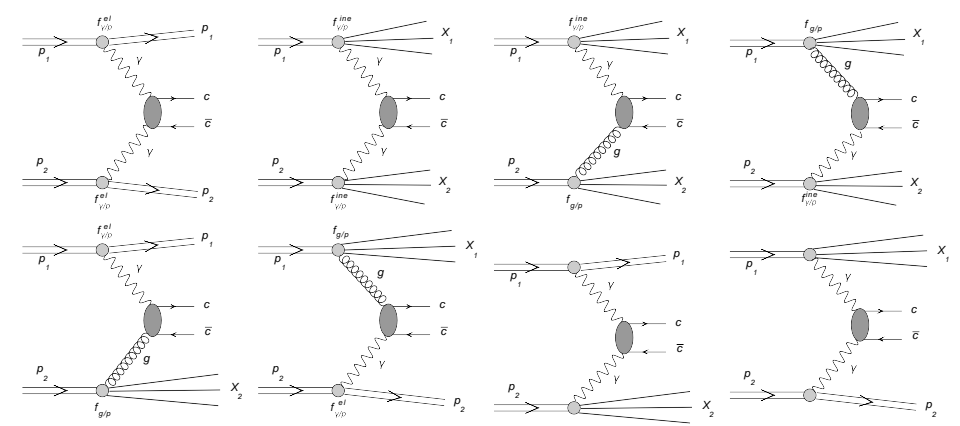

Figure 1: Diagrams representing the subleading mechanisms for production of heavy quarks discussed here.

\section{Production of heavy quarks}

The inclusive heavy quark/antiquark production can be calculated in the framework of the $k_{t^{-}}$ factorization. In this approach transverse momenta of initial partons are included and emission of gluons is encoded in so-called unintegrated gluon distributions (UGDFs) [1].

In the leading-order (LO) approximation within the $k_{t}$-factorization approach the quadruply differential cross section in the rapidity of $Q\left(y_{1}\right)$, in the rapidity of $\bar{Q}\left(y_{2}\right)$ and the transverse momentum of $Q\left(p_{1}, t\right)$ and $Q\left(p_{2}, t\right)$ can be written as

$$
\begin{array}{r}
\frac{d \sigma}{d y_{1} d y_{2} d^{2} p_{1, t} d^{2} p_{2, t}}=\sum_{i, j} \int \frac{d^{2} \kappa_{1, t}}{\pi} \frac{d^{2} \kappa_{2, t}}{\pi} \frac{1}{16 \pi^{2}\left(x_{1} x_{2} s\right)^{2}} \overline{\left|\mathscr{M}_{i j}\right|^{2}} \\
\delta^{2}\left(\vec{\kappa}_{1, t}+\vec{\kappa}_{2, t}-\vec{p}_{1, t}-\vec{p}_{2, t}\right) \mathscr{F}_{i}\left(x_{1}, \kappa_{1, t}^{2}\right) \mathscr{F}_{j}\left(x_{2}, \kappa_{2, t}^{2}\right)
\end{array}
$$

where $\mathscr{F}_{i}\left(x_{1}, \kappa_{1, t}^{2}\right)$ and $\mathscr{F}_{j}\left(x_{2}, \kappa_{2, t}^{2}\right)$ are the so-called unintegrated gluon (parton) distributions. The unintegrated parton distributions are evaluated at: $x_{1}=\frac{m_{1, t}}{\sqrt{s}} \exp \left(y_{1}\right)+\frac{m_{2, t}}{\sqrt{s}} \exp \left(y_{2}\right), x_{2}=\frac{m_{1, t}}{\sqrt{s}} \exp \left(-y_{1}\right)+$ $\frac{m_{2, t}}{\sqrt{s}} \exp \left(-y_{2}\right)$, where $m_{i, t}=\sqrt{p_{i, t}^{2}+m_{Q}^{2}}$. Some applications can be found also in Ref.[2].

\section{MRST-QED parton distributions}

The factorization of the QED-induced collinear divergences leads to QED-corrected evolution equations for the parton distributions of the proton [3].

$$
\begin{aligned}
\frac{\partial q_{i}\left(x, \mu^{2}\right)}{\partial \log \mu^{2}} & =\frac{\alpha_{S}}{2 \pi} \int_{x}^{1} \frac{d y}{y}\left\{P_{q q}(y) q_{i}\left(\frac{x}{y}, \mu^{2}\right)+P_{q g}(y) g\left(\frac{x}{y}, \mu^{2}\right)\right\} \\
& +\frac{\alpha}{2 \pi} \int_{x}^{1} \frac{d y}{y}\left\{\tilde{P}_{q q}(y) e_{i}^{2} q_{i}\left(\frac{x}{y}, \mu^{2}\right)+P_{q \gamma}(y) e_{i}^{2} \gamma\left(\frac{x}{y}, \mu^{2}\right)\right\} \\
\frac{\partial g\left(x, \mu^{2}\right)}{\partial \log \mu^{2}} & =\frac{\alpha_{S}}{2 \pi} \int_{x}^{1} \frac{d y}{y}\left\{P_{g q}(y) \sum_{j} q_{j}\left(\frac{x}{y}, \mu^{2}\right)+P_{g g}(y) g\left(\frac{x}{y}, \mu^{2}\right)\right\} \\
\frac{\partial \gamma\left(x, \mu^{2}\right)}{\partial \log \mu^{2}} & =\frac{\alpha}{2 \pi} \int_{x}^{1} \frac{d y}{y}\left\{P_{\gamma q}(y) \sum_{j} e_{j}^{2} q_{j}\left(\frac{x}{y}, \mu^{2}\right)+P_{\gamma \gamma}(y) \gamma\left(\frac{x}{y}, \mu^{2}\right)\right\} .
\end{aligned}
$$


Such a parton set, including photon as a parton in the proton, has been found in Ref.[3]. These parton distributions can be used to calculate several of the mechanism shown in Fig.1. The elastic contribuions shown in Fig.1 are calculated using photon distributions which can be calculated exclusively from QED including extended nature of the proton via electromagnetic form factors.

\section{Results}

As an example in Fig.3 we show contribution of the subleading mechanisms for the nominal, hopefully future, LHC energy $W=14 \mathrm{TeV}$. We show distribution in transverse momentum and rapidity.
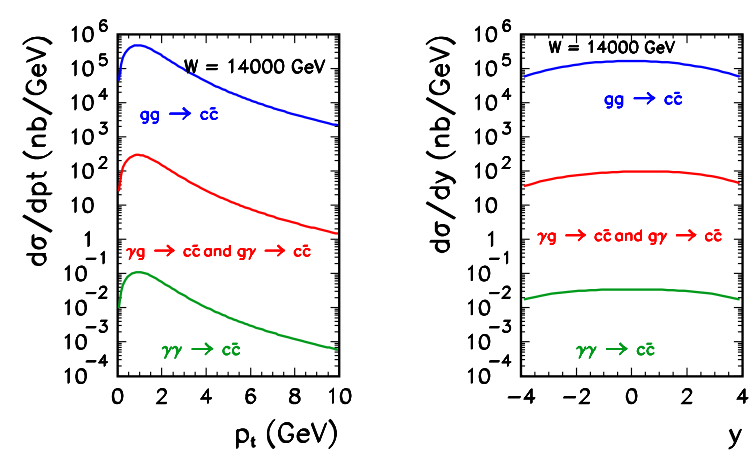

The mechanisms with photons give rather small contribution to the open charm production. For open bottom poroduction their contribution would be even smaller due to the smaller charge of the $b$ quark.

\section{References}

[1] M. Łuszczak and A. Szczurek, Phys. Rev. D73 (2006) 054028.

[2] M. Łuszczak, R. Maciuła, A. Szczurek, Phys. Rev. D79 (2009) 034009.

[3] A.D. Martin, R.G. Roberts, W.J. Stirling and R.S. Thorne, Eur. Phys. J C39 (2005) 155. 